ESJ Humanities

\title{
L'aplication de la Methode Indicametrique a l'Analyse des Projets et Programmes: Cas du Secteur Emploi-Formation en Cote d'Ivoire
}

\author{
Meite Inza \\ Economiste-Démographe, \\ Doctorant Université Internationale Ibéro-Américaine Mexico \\ Dr. Ruben Torres \\ Directeur de Thèse, Professeur-Chercheur en Sciences Politiques, Centre \\ Péninsulaire en Humanités Sciences Sociales (CEPHCIS) de l'Université \\ Nationale Autonome du Mexique (UNAM)
}

Doi:10.19044/esj.2021.v17n42p39

Submitted: 17 November 2021

Accepted: 02 December 2021

Published: 31 December 2021
Copyright 2021 Author(s)

Under Creative Commons BY-NC-ND

4.0 OPEN ACCESS

Cite As:

Inza M. \& Torres R. (2021). L'aplication de la Methode Indicametrique a l'Analyse des Projets et Programmes: Cas du Secteur Emploi-Formation en Cote d'Ivoire. European Scientific Journal, ESJ, 17 (42), 39. https://doi.org/10.19044/esj.2021.v17n42p39

\section{Résumé}

Le présent article s'inscrit dans le cadre de notre travail de recherche de thèse de doctorat en gestion de projets portant sur le thème : «Structure du marché du travail en Côte d'Ivoire : une analyse descriptive à la lumière des projets et programmes d'emploi ». A travers cette thématique, vous voulons contribuer à l'amélioration de l'employabilité et de l'insertion des personnes en âges de travailler, notamment les jeunes diplômés. Il s'agit également de promouvoir de nouveaux paradigmes et outils en matière de conception et d'analyse de risques de projets, en plus de ceux déjà existants. En effet, les faibles taux de chômage observés au cours des enquêtes emploi de ces cinq dernières années ( enquêtes emploi 2012, 2014, 2016, 2017 et 2019) voilent une résurgence des emplois informels et précaires au détriment des emplois formels. La population au chômage est majoritairement composée de primo demandeurs d'emploi, notamment les diplômés de l'enseignement technique, professionnel ainsi que de l'enseignement supérieur. Ce qui pose d'ailleurs, la question de l'efficacité externe du système national de formation, ainsi que des projets et programmes d'insertion. Pour ce faire, nous avons jugé 
judicieux de passer en revue une série de treize (13) projets mis en œuvre ou en cours de réalisation, en nous servant des outils de la science indicamétrique. Cette discipline a la particularité de mettre en exergue les facteurs susceptibles d'engendrer la performance, l'efficacité et l'efficience d'un projet, en se basant sur les capacités intrinsèques des personnes physiques et morales impliquées dans sa gestion. Au plan méthodologique, nous avons recours à un des outils importants de la science en indicamétrie : la carte capacitaire. Elle comprend un ensemble d'indicateurs qui s'expriment en Valeur Intrinsèque Capacitaire Energétique (VICE) normée. Toute valeur en dessous de la norme est considérée insuffisante ou en déficit. Toute valeur au-dessus de la norme est dite, en excédent. Ainsi, à l'issue des différentes analyses, nous avons abouti aux principaux résultats suivants: (i) la prise en compte des caractéristiques de l'environnement interne et externe des projets accroit leur probabilité de succès, (ii) le choix de la dénomination de tout projet ou programme influence son mode de gestion et (iii) la prise en compte des caractéristiques et des capacités intrinsèques des promoteurs ou gestionnaires des projets dans leur mise en œuvre accroit significativement leur probabilité de succès.

Mots-clés : Emploi, gestion de projets, indicateurs objectifs, système capacitaire, système capacitaire intrinsèque globalisante, science indicamétrique

\title{
Application of the Indicametric Method to the Analysis of Projects and Programs: Case of the Employment-Training Sector in Cote d'Ivoire
}

\author{
Meite Inza \\ Economiste-Démographe, \\ Doctorant Université Internationale Ibéro-Américaine Mexico \\ Dr. Ruben Torres \\ Directeur de Thèse, Professeur-Chercheur en Sciences Politiques, Centre \\ Péninsulaire en Humanités Sciences Sociales (CEPHCIS) de 1'Université \\ Nationale Autonome du Mexique (UNAM)
}

\begin{abstract}
This paper is part of the research work for a doctoral thesis in project management titled "Structure of the labor market in Côte d'Ivoire: a descriptive analysis in the light of projects and programs of use". The objective of this theme is to improve the employability and integration of people of
\end{abstract}


working age, especially young graduates. It also focuses on promoting new paradigms and tools for project design and risk analysis, in addition to existing ones. The low unemployment rates observed during employment surveys over the past five years veil a resurgence of informal and precarious jobs to the detriment of formal jobs. The unemployed population is mainly made up of first-time job seekers, particularly graduates of technical, vocational, and higher education. This raises the question of the external effectiveness of the national training system as well as the integration projects and programs. To answer this question, a series of thirteen (13) projects that were either implemented or in progress was reviewed through the tools of indicametric science. This discipline has the particularity of highlighting the factors that are likely to generate the performance, effectiveness, and efficiency of a project based on the intrinsic capacities of the natural and legal persons involved in its management. As for the methodology, there is recourse to one of the important indicametry tools: the capacity card. It includes a set of indicators which are expressed in standardized Intrinsic Energy Capacitance Value (VICE). Any value below the standard is considered insufficient or in deficit, while any value above the norm is said to be in excess. At the end of the various analysis, the following main results have been achieved: (i) taking into account the characteristics of the internal and external environment of projects increases their probability of success, (ii) the choice of name of any project or program influences its mode of management and (iii) taking into account the characteristics and intrinsic capacities of the promoters or managers of projects in their implementation significantly increases their probability of success.

Keywords: Employment, project management, objective indicators, capacity system, intrinsic capacity system, globalizing, indicametric science

\section{Introduction}

\section{a. Contexte de l'étude}

L'importance des problèmes socio-humains, économiques et financiers n'échappe plus aujourd'hui à personne. Tout le monde reconnaît que c'est de leur résolution que dépendent non seulement le bien-être matériel et moral des peuples mais aussi la paix en général.

Il importe donc, que la connaissance des principaux problèmes et mécanismes socio-économiques cesse d'être le domaine réservé de quelques spécialistes et deviennent l'apanage de tout citoyen éclairé. D'ordinaire, les grands économistes et financiers se protègent du monde extérieur en adoptant un langage exotérique ou un discours hermétique pour le non initié. Les sciences ont, de façon générale pour objet de dégager des généralités récurrentes, de définir des concepts. Les lois et les systèmes commencent 
toujours par la reconnaissance des rapports préexistants pour s'en écarter insidieusement et poser des absolus à priori. Ce que Kuhn appelle des « paradigmes ${ }^{1} »$.

Il existe donc "une sorte de réalité “indépendante", c'est-à-dire qui ne peut être appréhendée en l'état actuel de nos outils d'observation sensibles et conceptuels » (http://fr.wikipedia.org/wiki/Ph\%C3\%A9nom\%C3\%A8ne). Cette « méta réalité » selon certains scientifiques classiques, n'appartient pas aux domaines étudiés par la science, et bien, qu'influençant notre quotidien, elle ne saura jamais se rendre perceptible. Ainsi, plusieurs théories cognitives ont été élaborées pour déchiffrer et balayer l'espace qui va du perçu à l'intelligible, au connaissable : comment passe-t-on du phénomène à l'objet, de l'observation à la déduction de lois générales ? Si la connaissance interroge les phénomènes, elle interroge peut-être surtout, à travers eux, notre vision du monde, c'est-à-dire les relations entre un « sujet » et un « objet ». Les sciences humaines mesurent donc la dimension de l'esprit humain et prennent le pas sur des réflexions auparavant dévolues à la philosophie. S'il est vrai que l'homme est au début, au centre et à la fin de tout processus de développement, nombreux sont les auteurs éminents qui ont déjà écrit sur les concepts du développement depuis les préclassiques jusqu'aux contemporains en passant par les classiques et les néoclassiques. Mais très peu d'entre eux ont insisté sur son aspect capacitaire.

Depuis les penseurs comme Karl MAX, Emile DURKHEIM, Max WEBER, David RICARDO ou Adam SMITH, on s'intéresse aux fondements politiques et sociaux de l'économie, aux différents mécanismes de régulation des politiques du marché pour rendre compte des transformations des sociétés. Tous ces rapports sociaux peuvent être pris dans leur aspect numérique ; et, dans ces conditions s'inscrivent dans des statistiques ${ }^{2}$.

Ainsi, la gestion (humaine / financière) des projets voire de l'entreprise est un domaine en constante évolution et des approches différentes ou nouvelles sont proposées pour saisir les flux qui ont comme origine ou comme destination l'entreprise.

Ces approches conduisent en général à privilégier un ou plusieurs indicateurs dont l'évolution est un élément du diagnostic sur la qualité d'une gestion.

En effet, comment pourrait-on prétendre réaliser un quelconque objectif majeur du développement, quand des populations ne maitrisent pas leur système capacitaire permettant ainsi d'orienter, de démocratiser en vue d'autoriser l'épanouissement individuel et collectif, et d'éviter de créer des déchets humains ou des marginaux sociaux. Selon le Professeur Moustapha DIABATE (1999), fondateur de l'indicamétrie, « l'étude du cerveau et de ses

${ }^{1}$ T. Kuhn, La structure des révolutions scientifiques, 1962

${ }^{2}$ Delas Jean-Pierre et Milly Bruno. «Histoire des pensées sociologiques », Paris. Dalloz, 1997. 
capacités doit permettre de construire de nouveaux indicateurs humains et socio-humains. Il s'agira d'aller au-delà des indicateurs classiques ou objectifs (BIO) relevant et mesurant l'impact du visible pour mesurer désormais l'impact de tout ce qui est invisible sur l'Homme, sa communauté et son environnement global. Ce qui suppose qu'à la technologie de la musculature ou du travail physique que l'Afrique a connu depuis la traite négrière, il faut ajouter celle du cerveau et de ses capacités, condition essentielle pour bâtir une Afrique majeure dans un monde majeur».

De ce qui précède nous allons, dans les sections à venir, mettre en exergue la pertinence de l'indicamétrie, tout en mettant en exergue ses aspects innovants en matière de conception et de gestion de projets.Plus précisément, il s'agira pour nous d'appliquer la méthode indicamétrique à l'analyse de faisabilité de quelques projets et programme initiés par les gouvernements successifs en Côte d'Ivoire depuis plusieurs années, par l'entremise des ministères en charge de l'emploi, de la formation, de la jeunesse et des affaires sociales.

\section{b. Justification et objectifs de l'étude}

La situation d'occupation de la population en âge de travailler en Côte d'Ivoire porte les marques des différentes crises socio-politique et politicomilitaire qu'à connu le pays depuis deux décennies. Le niveau d'emploi a baissé alors que la population ivoirienne s'est accrue. Les faibles niveaux de taux de chômage observés au cours des enquêtes emploi de ces cinq dernières années ( Enquêtes emploi 2012, 2014, 2016, 2017 et 2019) voile une résurgence des emplois informels et vulnérables ( environ 94\% des emplois dans le secteur informel, selon l'Enquête Emploi 2019) au détriment des emplois formels. La population au chômage est majoritairement composée de primo demandeurs d'emploi, notamment les diplômés de l'enseignement technique, professionnel ainsi que de l'enseignement supérieur. Ce qui pose d'ailleurs, la question de l'efficacité externe du système national de formation D'une manière générale, cette étude visait à contribuer à l'amélioration de l'efficacité et l'efficience des projets et programmes, notamment dans le secteur emploi-formation.

De façon spécifique, il s'agira de :

- Identifier les facteurs subjectifs de risques liés à la conception, la gestion et l'impact des projets et programmes ;

- Proposer des indicateurs subjectifs et mesurables pour l'analyse des projets et programmes ;

- Identifier les facteurs déterminants de succès des projets et programmes ;

- Identifier les caractéristiques intrinsèques individuelles des promoteurs et gestionnaires de projets et programmes. 
Notre procéderons à l'analyse-diagnostic capacitaire des projets initiés en faveur de la jeunesse en rapport avec le système capacitaire révélé par les études d'identité capacitaire intrinsèque des projets concernés.

Pour ce faire, un rappel des paradigmes, notamment des fondements théoriques et méthodologique de la science indicamétrique s'avère nécessaire, avant la présentation des résultats et éventuellement des propositions de recommandations.

\section{Fondements théoriques et méthodologie d'approches}

\subsection{Brève historique de l'indicamétrie}

L'indicamétrie a été inventée par l'ivoirien, le Professeur Moustapha DIABATE depuis 1963, après avoir analysé les critiques faites par des observateurs occidentaux sur les pays africains accédant nouvellement à leur indépendance. Il s'agit notamment des propos du journaliste Gilbert COMTE qui disait un jour en conférence à Paris que tout se passait comme si la jeunesse africaine et ses dirigeants étaient atteints d'une incapacité congénitale à résoudre leurs problèmes (problèmes liés à la gestion des affaires). Celui-ci formulait son observation dans un contexte marqué une série d'instabilités sociales et politiques dans la plupart de ces pays africains, notamment en Afrique de l'ouest. Cette assertion a amené le Professeur DIABATE à débuter ses travaux de recherche sur la thématique "capacité et développement ${ }^{3}$ ». Toutefois, pendant son parcours, l'Indicamétrie fut considérablement enrichie grâce aux débats internationaux dont nous citerons quelques-uns:

$>$ Le Congrès de la fédération mondiale des études sur le futur en 1979 à Berlin (Allemagne),

$>$ Le Colloque international de l'UNESCO en 1979 à Athènes (Grèce),

$>$ Le séminaire international sur la planification en 1979 à Stamford (Etats-Unis d'Amérique),

$>$ Le Symposium des Nations Unies sur la science et la technique en 1979 à Paris (France),

$>$ Le Congrès mondial de la prospective sociale (Dakar - 1980),

> Plusieurs invitations dans les universités américaines (1980) et centres de recherche au Canada - Québec (Montréal 1979-1980),

> L'Atelier International de l'UNESCO sur les Indicateurs socioéconomiques de novembre 1981 tenu à Abidjan (Côte d'Ivoire),

$>$ Le Séminaire international sur les manuels de la planification en 1983 à Rabat (Maroc),

$>$ le Forum de la presse du Tiers monde en 1983 à Dakar (Sénégal),

$>$ Congrès mondial sur les modèles économiques organisé par l'UNESCO en 1984 à Buenos Aires (Argentine).

${ }^{3}$ Thème de recherche pour thèse de doctorat paris 1953 
Ces différents débats vont contribuer à propulser l'indicamétrie au-devant de la scène nationale et internationale, notamment dans le monde scientifique et intellectuel, à travers une série d'évènement dont les principaux sont cités cidessous :

> L'organisation africaine de Propriété Intellectuelle (OAPI), par lettre datée du 24 février 1983, reconnaît que l'Indicamétrie est une découverte scientifique qui mérite d'être enregistrée au niveau de l'Accord international sur les découvertes à Berne (Suisse) ;

$>$ En 1994, le Conseil International de l'Indicamétrie (CIDI), est créée à Dakar (Sénégal) sous l'autorisation $\mathrm{N}^{\circ} 000101 / \mathrm{MINT} / \mathrm{DAGAT} / \mathrm{DEL} / \mathrm{AS}$ du 10 janvier 1994 avant la création à Ouidah (Bénin) d'un département de recherche précédent celui du département d'Indicamétrie de l'Université de Bouaké (Côte d'Ivoire) en janvier 1998 ;

$>$ En août 1996, l'UNESCO -BREDA et le Ministère de l'Education Nationale du Sénégal organisent la $1^{\text {ère }}$ journée internationale sur l'Indicamétrie ;

$>\mathrm{Au}$ colloque international d'Ouidah de 2003 sur le thème «Forces porteuses des Diasporas Africaines, Stratégies pour un développement pertinent et sûr de l'Afrique ", l'Indicamétrie est considérée comme la science la plus appropriée pour le développement de l'Afrique ;

$>$ Le 9 septembre 2004 à l'Hôtel du Golf à Abidjan (Côte d'Ivoire), la Primature à travers le Secrétariat National à la Gouvernance et au Renforcement des Capacités parraine la cérémonie de remise des diplômes aux apprenants professionnels en Indicamétrie. Après cette cérémonie, les autorités de l'Université de Bouaké décident de transformer le Département d'Indicamétrie en Institut Multipolaire d'Etude de Recherche et de Formation en Indicamétrie (IMERFI).

\subsection{Fondements théoriques}

Le concept pivot de cette science est celui de l'invisible, la subjectivité, avec son corollaire, la capacité. L'invisible s'entend communément comme ce qui n'est pas visible par nos sens voire par nos pensées. En plus de cette assertion courante de l'invisible, le concept de l'invisible signifie ce qui est dans (« in »). En d'autres termes, l'invisible reste inaccessible aussi longtemps que par nos efforts, notre intelligence, notre volonté de découvrir, ce qui dans le visible n'est pas visible. Ainsi, l'être humain confronté à de l'invisible va innover, créer, faire preuve d'ingéniosité et imaginer des outils concrets ou virtuels pour rendre visible cet invisible. Il en est ainsi des statistiques qui, en se servant des outils mathématiques, consacrent les sondages pour accéder à l'invisible. Il en est de même des sciences informatiques avec ses algorithmes invisibles qui sous-tendent les informations visibles. Ces efforts de 
compréhension de la globalité d'une réalité se retrouvent aussi en Sciences économiques où l'économétrie tente d'appréhender la réalité économique dans toute sa globalité. Toutes les sciences déploient des efforts et des moyens pour circonscrire l'invisible. Toutefois, en dépit de tous ces efforts, ces disciplines peinent à couvrir ou à appréhender toute la réalité dans sa globalité en intégrant l'invisible (Science de l'indicamétrie, cours chrono profil : activités et familles énergétiques, Serge , 2018)

En ce qui concerne la notion de « capacité » érigée en concept scientifique par la science indicamétrie, elle est intimement liée au concept de " l'invisible ». La capacité admet, désormais, grâce à l'indicamétrie " une définition précise et, elle est mesurable, comme l'est la masse en physique, le triangle en géométrie et l'intensité du courant en électricité. » (A. Deazon, In introduction à l'indicamétrie, pp 43).

Il était devenu nécessaire pour la science indicamétrique de clarifier la notion de capacité (Cours postulat de base, 2018). Jusque-là, la capacité a indiqué, tantôt un contenant de connaissances exprimé par «la capacité de la mémoire à long terme » de P. Astolfi (1998, pp 106-107, cité par A. Deazon, 2011), tantôt un contenu de connaissances défini comme «... des capacités et de compétences, qui rendent les savoirs transférables et mobilisables dans des situations professionnelles » de Ph. Perrenoud, (cité par A. Deazon pp 43).

En définitive, en science indicamétrique, la capacité, ramenée à l'être humain, à tout existant, indique l'énergie qui fait que l'habileté de celui-ci s'exprime et que sa compétence se mobilise.

En l'absence de cette énergie rien ne se produirait, cette dose d'énergie doit être suffisante pour activer le succès ou l'efficacité de tout projet.

Le concept de capacité devenu la pierre angulaire dans la procédure de conception et de gestion des projets se retrouve de façon systématique dans la formulation du contenu des projets en conformité avec la prise en compte de tous les paramètres afférents à ceux-ci. Ainsi, l'on s'est habitué, en matière de conception du contenu des projets, à des expressions comme : «à la fin du processus, le projet doit être capable de générer des emplois... », ou « le projet devrait impacter positivement la jeunesse. ».

En outre, la science indicamétrique, en se dotant des outils pour capter, quantifier et mesurer la dose d'énergie nécessaire à la réalisation de toute chose, pourrait contribuer à détecter, bien avant même tout démarrage du processus, les facteurs de succès ou d'échec desdits projets.

Ainsi, les mesures à prendre seront envisagées et la structure ou l'institution à l'origine du projet gagnerait en temps et en efficacité. Cela, éviterait la reprise du schéma de l'analyse systémique sous forme de schéma rétroaction.

Cependant, il est nécessaire, qu'avant le démarrage du projet les cartes capacitaires du projet, de l'initiateur, du chef et du gestionnaire du projet ainsi 
que celle du pays, de la ville ou de la localité dans laquelle le projet sera exécuté, puissent être élaborées.

En effet, la science de l'indicamétrie propose une approche holistique, afin d'ajuster les prises de décisions et permettre aux différents acteurs de la chaine de faire des choix efficients. Il en résultera donc une affectation judicieuse et rationnelle des ressources aux différents projets et programmes.

La science indicamétrique intervient dans tous les domaines et permet d'identifier de façon efficace et efficiente, les atouts, les faiblesses, les menaces et les possibilités liés au projet et à son environnement d'exécution. Pour y parvenir, l'indicamétrie s'appuie sur un outil d'analyse assez pertinent, à savoir : la Carte d'Identité Capacitaire Intrinsèque (CICI).

Dès lors, il devient possible et aisé pour le chercheur de comprendre les différences de succès dans la réalisation de différents projets et voir dans quelle mesure prendre en compte ces déterminants dans la conception de projets futurs.

En définitive, l'Indicamétrie vient combler le vide entre les deux pôles entre lesquels oscillent les sciences sociales dans leur quête de «statut scientifique », sans jamais y parvenir, à savoir, le pôle «normatif » et le pôle « indicatif » .

Selon le professeur DIABATE, fondateur de la science indicamétrique, cette discipline est fondée sur la double considération suivante :

- d'une part, ni la science économique, ni les autres disciplines des sciences humaines (disciplines infructueuses), ne prennent en compte toutes les dimensions de l'homme, et de la société ;

- et que d'autre part, le fait pour les sciences sociales, de tendre vers le model des sciences dites exactes (disciplines fructueuses), par la mesure et la quantification, n'y suffit pas tant que l'on ignore les dimensions classées comme subjectives, irrationnelles, parce que non saisissables dans les limites étroites des critères prédéfinis de «l'objectivité scientifique » de l'heure.

\subsection{Méthodologie d'approche}

Cette section est dédiée à la présentation de la méthodologie d'approche pour l'analyse des projets et programmes concernés. Plus précisément, il s'agira, notamment de présenter le cadre et la délimitation de l'étude, d'exposer les techniques de collecte des données, la technique de l'échantillonnage, la mise en œuvre de l'outil de recherche, le dépouillement, la méthode d'analyse et les difficultés rencontrées.

\subsubsection{Population et échantillonnage des groupe-sujets}

L'analyse porte sur un échantillon de 13 projets et programmes d'insertion pertinents, notamment en faveur des jeunes et autres personnes 
vulnérables. Ces projets sont menés par diverses structures ministérielles. Certains projets sont clos tandis que d'autres sont en cours. La liste des projets et programmes a été obtenue à travers une revue de documents de politiques publiques d'emploi, de rapports d'analyse situationnelle du secteur éducation/formation et d'articles scientifiques sur la thématique de l'emploi en Côte d'Ivoire. Pour les besoins de l'étude, nous avons décidé de n'étudier que les projets et non les gestionnaires de ceux-ci.

Le tableau suivant met en évidence les treize (13) projets.

Tableau 1. Liste des projets et programmes de l'étude

\begin{tabular}{|c|c|c|}
\hline $\mathrm{N}^{0}$ & INTITULE DU PROJET & CODES \\
\hline 1 & $\begin{array}{l}\text { Projet d'Appui à la Formation de la Population Active } \\
\text { (PAFPA) }\end{array}$ & PAFPAY03 \\
\hline 2 & Le projet «Gérez Mieux votre Entreprise» (GERME) & PGMVEY05 \\
\hline 3 & $\begin{array}{l}\text { Le Projet de Formation par Apprentissage (PFA) } \\
\text { dans le cadre du Programme d'Absorption des Jeunes } \\
\text { Déscolarisés (PAJD) }\end{array}$ & PFA/AJDY06 \\
\hline 4 & $\begin{array}{l}\text { Le Projet d'Appui et d'Accès des Personnes Handicapées à la } \\
\text { Formation Professionnelle (PAAPHFP) }\end{array}$ & P2APHFCY9 \\
\hline 5 & Le Programme d'Aide à l'Embauche (P.A.E.) & P2AY10 \\
\hline 6 & $\begin{array}{l}\text { Le Fonds de soutien à l'emploi par les travaux d'utilité } \\
\text { publique à haute intensité de main d'œuvre (FSE-THIMO) }\end{array}$ & FSETHIMOY11 \\
\hline 7 & $\begin{array}{l}\text { Le Projet d'Appui au Traitement Economique du Chômage } \\
\text { (PATEC) }\end{array}$ & PATECY12 \\
\hline 8 & $\begin{array}{l}\text { Projet Emploi des Jeunes et Développement des } \\
\text { Compétences (PEJEDEC) }\end{array}$ & PEJDCY13 \\
\hline 9 & $\begin{array}{l}\text { Programme spécial de requalification et de mise en } \\
\text { adéquation emploi-formation }\end{array}$ & PSRMAEFY14 \\
\hline 10 & $\begin{array}{l}\text { Service civique d'aide à l'emploi et au développement } \\
\text { (SCAED) }\end{array}$ & SCAEDY17 \\
\hline 11 & $\begin{array}{l}\text { Programme d'Appui à l'Amélioration de l'Employabilité et à } \\
\text { l'Insertion des Jeunes (PAAEIJ) volet THIMO }\end{array}$ & P2AEIJY19 \\
\hline 12 & C2D Emploi & C2DEY20 \\
\hline 13 & Agir pour les jeunes & APJY21 \\
\hline
\end{tabular}

Source : Auteur, codage des projets dans la phase d'analyse

\subsubsection{Technique de collecte et de traitement des données e}

Nous avons recours à un des outils importants de la science en indicamétrie, la carte capacitaire. C'est un outil qui révèle les capacités ou valeurs intrinsèques de tout phénomène, de tout projet étudié afin de déterminer les atouts et faiblesses de ceux-ci.

Les indicateurs des capacités les plus usuelles sont au nombre de cinquante-sept (57) environ et peuvent se regrouper en quatre catégories, les capacités de la famille énergétique, les capacités intellectuelles, humaines, de réalisation ou d'activation des capacités, financières ou de richesse et les capacités complémentaires. 
Toutes ces capacités s'expriment en VICE (valeur intrinsèque capacitaire énergétique). A chaque capacité est affecté un code, au format, Yn. Elles sont circonscrites dans un tableau spécifique, le matriciel stratégique qui présente les valeurs obtenues au regard de leurs normes. Toute valeur en dessous de la norme est considérée insuffisante ou en déficit. Toute valeur au-dessus de la norme est dite, en excédent.

Nous avons retenu 13 projets dont nous étudions la carte capacitaire. Il n'est envisagé aucun questionnaire, aucune interview à soumettre ni aux promoteurs, ni aux structures chargées de les conduire, ni aux bénéficiaires. L'étude à proprement parler va suivre les six (6) étapes ci-dessous décrite :

\section{L'apprêt de la thématisassions du sujet}

Il consiste à relever l'identité du projet en retenant que la dénomination dudit projet, tel que figurant sur les documents d'identification. C'est l'apprêt de la thématisassions.

\section{Transformation chiffrée de la thématisassions}

L'apprêt du sujet est transformé en chiffres par l'Alphabet Indicamétrique (A.I). A chaque lettre contenu dans la dénomination du projet, est affecté un chiffre allant de 1 à 9 .

\section{Fréquence initiale des nucléaires de socle}

On détermine en suite, sur l'échelle des nucléaires de socle que sont 1 , $2,3,4,5,6$ et 7 , les fréquences des chiffres issues de la numérisation par l'alphabet indicamétrique de l'identité du sujet.

\section{Fréquence définitive des nucléaires de socle}

Ensuite, on établit les fréquences définitives qui consistent à attribuer aux valeurs absentes des fréquences additionnelles à partir des moléculaires produites par la numérisation de l'identité du sujet.

\section{Matricielle des valeurs, Yn.}

Toutes ces fréquences obtenues vont être utilisées dans un logiciel spécifique en vue de produire une matricielle des valeurs capacitaires.

\section{Extraction des indicateurs pour le choix des projets}

Pour les besoins de l'étude nous retenons des indicateurs spécifiques au choix des projets afin de retenir ceux qui pourront cadrer avec les objectifs des promoteurs dans le cadre de l'insertion de la jeunesse. 


\subsubsection{Analyse de la carte capacitaire ou Moyens d'évaluation}

Dans un premier temps, nous établissons la matricielle stratégique de chaque sujet. A partir de cette matricielle, nous identifions, selon les normes de l'indicamétrie, les forces et faiblesses de chaque indicateur de chaque projet. Nous relevons ensuite les valeurs des 10 indicateurs pour le choix des projets à retenir selon le tableau suivant.

Tableau 2. Matricielles stratégiques pour l'analyse d'un projet

\begin{tabular}{|c|c|r|r|r|r|r|r|r|r|r|r|r|}
\hline ICH & Y13 & Y14 & Y15 & Y16 & \multicolumn{1}{|c|}{ Y6 } & \multicolumn{1}{|c|}{ Y19 } & Y20 & Y22 & Y24 & Y25 & \multicolumn{1}{|c|}{ Y26 } & Y28 \\
\hline Norme & $+, 0,-$ Yn & 3,75 & 0,75 & 10 & 100 & 100 & 30 & 1 & 50 & 0,75 & 0,5 & 1 \\
\hline
\end{tabular}

\begin{tabular}{|c|c|r|c|r|r|r|r|r|}
\hline ICH & Y30 & Y39 & Y40 & Y41 & Y42 & Y43 & Y44 & Y45 \\
\hline Norme & 5000 & 1 & 22,75 & 3,75 & 2 & 1,5 & 15 & 50 \\
\hline
\end{tabular}

Source : cours d'analyse indicamétrique, Prof. DIABATE Moustapha

Tableau 3. Présentation des Indicateurs d'évaluation de la faisabilité de projets : déterminants standards d'efficacité

\begin{tabular}{|c|c|}
\hline CODES & SIGNIFICATIONS \\
\hline Y13 & $\begin{array}{l}\text { FAMILLE ENERGETIQUE } \\
\checkmark \quad \text { la famille [+] (plus) symbolise l'énergie promotionnelle, conservatrice, } \\
\text { ascensionnelle du bâtisseur de stratégies et de modèles. C'est la famille } \\
\text { d'énergie réformatrice } \\
\checkmark \quad \text { la famille [0] (chiffre zéro) symbolise l'énergie centralisatrice, } \\
\text { décentralisatrice } \\
\checkmark \quad \text { la famille [-] (moins) symbolise l'énergie transformatrice, créatrice, } \\
\text { fondatrice (Tout terrain) } \\
\underline{\mathrm{NB}} \text { : Déterminer les capacités motrices et stratégiques permettant de mettre en } \\
\text { œuvre le message fondateur conditionnant la réussite (vie personnelle ou } \\
\text { projet). }\end{array}$ \\
\hline Y14 & $\begin{array}{l}\text { Le [Y14] dont la norme est } 3,75 \text { indique la puissance du mobilisateur d'énergie. } \\
\text { Cette capacité intrinsèque comprend le démarreur, l'accélérateur, le multiplicateur } \\
\text { et le distributeur d'énergie (DAME) de l'enseignant dont la famille d'énergie est } \\
\text { promotionnelle et/ou transformatrice }\end{array}$ \\
\hline Y20 & $\begin{array}{l}\text { Le [Y 20] dont la norme est } 30 \text { est l'endurance, la force de frappe et la persévérance } \\
\text { du sujet } \\
\text { ENDURANCE -VIABILTE }\end{array}$ \\
\hline Y24 & TECHNICITE - ANALYSE DE RISQUES DIVERS \\
\hline Y16 & $\begin{array}{l}\text { COMPÉTITION -ADVERSITÉ - FLUX AUTORITE - GESTION CONFLITS } \\
\text { ET TENSION LIEE A L'ENVIRONNEMENT }\end{array}$ \\
\hline Y25 & $\begin{array}{l}\text { Le [Y 25] est le stabilisateur d'une situation individuelle ou d'une entreprise en } \\
\text { difficulté 'effet de compétition, de rivalités ou tentatives de déstabilisation. Sa } \\
\text { norme est } 0,75 \\
\text { STABILISATION-DESTABILISATION }\end{array}$ \\
\hline Y30 & POTENTIEL FINANCIER-RICHESSE MATERIEL \\
\hline Y39 & BONNE GOUVERNANCE-SAINE GESTION \\
\hline Y40 & HUMANISME-UTILITAIRE \\
\hline Y42 & PROBITE MORALE \\
\hline
\end{tabular}

Source : cours d'analyse indicamétrique, Prof. DIABATE Moustapha 
Dans un second temps, les indicateurs sont analysés selon le tableau des catégories de performances capacitaires afin de choisir le ou les projets censés correspondent ou donner satisfaction dans le cadre de la lutte contre le chômage de la jeunesse. Autrement dit, les scores des indicateurs permettront de révéler les atouts et les faiblesses des profils des projets. Ainsi donc, les projets dont les profils présenteront un minimum de trois déficits parmi les 10 indicateurs ne seront pas retenus, car ils présentent de gros risques pour ne pas atteindre les objectifs visés. Tous les indicateurs déficitaires seront mis en rouge.

La section suivante présente d'une part le dépouillement des données, les résultats et les analyses, d'autre part les propositions ou les recommandations.

\section{Présentation des résultats}

Nous présentons ci-dessous les résultats des treize (13) projets.

Les résultats issus des différentes cartes capacitaires des projets respectifs mis en annexe, sont décrits dans les tableaux ou matricielles stratégiques ci-dessous.

Les scores des indicateurs mis en rouge sont ceux qui se signalent par leurs faiblesses vis-à-vis de la norme, et en bleu les projets qui présentent beaucoup d'atouts et par conséquent offrent une garantie en termes de réalisation.

Les analyses révèlent que sur les 13 projets étudiés, 8 présentent un profil avec les atouts de réalisation sans risques majeurs, par contre les 5 autres affichent un profil dont le système présente en son sein des faiblesses. Les faiblesses inhérentes aux projets concernés ne sauront leurs permettre d'être viables, les risques de non réalisation étant très élevés.

De ce qui précède, nous allons d'une part présenter dans deux tableaux distincts les deux catégories de projets, c'est à dire les cinq (5) dont le risque de réalisation est élevé, soit 38,5\% et les huit (8) dont la faisabilité est fiable, soit $61,5 \%$; et d'autres parts, faire le diagnostic ou l'interprétation de ces résultats afin de révéler les causes profondes et subjectives qui ont permis de classer ces 13 projets en deux catégories, selon qu'ils soient réalisables ou non. 
Tableau 4. Matricielles stratégiques des 13 projets

INDICATEURS DE CAPACITES HUMAINES ET NON HUMAINES/ICH

\begin{tabular}{|c|c|c|c|c|c|c|c|c|c|c|}
\hline \multirow[b]{2}{*}{ CODES-PROJETS } & \multicolumn{10}{|c|}{ INDICATEURS DE CAPACITES HUMAINES ET NON HUMAINES/ICH } \\
\hline & Y13 & Y14 & Y16 & $\mathrm{Y} 20$ & $\mathrm{Y} 24$ & $\mathrm{Y} 25$ & $\mathrm{Y} 30$ & $\mathrm{Y} 39$ & $\mathrm{Y} 40$ & Y42 \\
\hline PAFPAY03 & $+45,00$ & 3,69 & 4,03 & 29,48 & 68,71 & $\mathbf{1 , 0 7}$ & 11154,32 & 4,71 & 22,19 & 5,33 \\
\hline PGMVEY05 & $+3,75$ & 3,38 & 0,39 & 27,04 & 69,63 & 0,09 & $\mathbf{9 5 9 9 , 5 3}$ & 2,14 & 20,35 & $\mathbf{4 , 2 0}$ \\
\hline PFA/AJDY06 & $-138,75$ & $\mathbf{3 , 8 8}$ & 6,57 & 31,08 & $72 ? 29$ & $\mathbf{3 , 3 0}$ & 26122,32 & 8,14 & 23,39 & 10,63 \\
\hline P2APHFCY9 & $-48,75$ & 4,00 & 2,88 & 32,04 & 78,27 & 1,16 & 21911,94 & 8,14 & 24,11 & 8,78 \\
\hline P2AY10 & $+41,25$ & 4,17 & 5,81 & 33,34 & 130,60 & $\mathbf{0 , 9 8}$ & 7104,24 & $\mathbf{3 , 0 0}$ & 25,09 & 3,84 \\
\hline FSETHIMOY11 & $+48,75$ & 3,85 & 2,40 & $\mathbf{3 0 , 7 8}$ & 63,74 & 1,16 & 20346,67 & 7,29 & 23,16 & 10,14 \\
\hline PATECY12 & $+26,25$ & 3,64 & 2,15 & 29,09 & 64,08 & 0,63 & 12215,29 & 4,29 & 21,8 & 5,76 \\
\hline PEJDCY13 & $+56,25$ & 3,72 & 4,28 & 29,80 & 115,83 & 1,34 & 13151,61 & 5,14 & 22,42 & 6,35 \\
\hline PSRMAEFY14 & $+67,50$ & 3,84 & 4,39 & 30,74 & 51,46 & 1,61 & 15372,38 & 7,71 & 23,13 & $\mathbf{7 , 8 5}$ \\
\hline SCAEDY17 & $\begin{array}{r}+75,00 \\
\end{array}$ & $\mathbf{3 , 8 5}$ & 6,46 & 30,83 & 91,15 & 1,79 & 11614,46 & $\mathbf{3 , 4 3}$ & 23,18 & 5,80 \\
\hline P2AEIJY19 & $+56,25$ & 3,82 & 3,56 & 30,58 & 61,64 & 1,34 & 15944,99 & 8,14 & 23,01 & 7,90 \\
\hline C2DEY20 & +60 & $\mathbf{3 , 8 2}$ & 4,45 & 30,56 & 156,13 & 1,43 & 13483,65 & 6,86 & 22,99 & 6,67 \\
\hline APJY21 & $+60,00$ & 3,01 & 9,63 & 24,06 & 44,74 & 1,43 & \begin{tabular}{|l|l|}
6230,98 \\
\end{tabular} & 1,71 & 18,19 & 2,43 \\
\hline NORMES & $+, 0,-$ & 3,75 & 10 & 30 & 50 & 0,75 & 5000 & 1 & 22,75 & 2 \\
\hline
\end{tabular}

\subsection{Analyse des projets à faibles niveaux de faisabilité}

Dans le souci de mieux faire ressortir les faiblesses de ces différents projets, nous effectuerons des analyses diagnostics par vague d'indicateurs.

Tableau 5. Matricielle stratégique des 5 projets non réalisables

\begin{tabular}{|c|c|c|c|c|c|c|c|c|c|c|}
\hline \multirow[b]{2}{*}{ CODES-PROJETS } & \multicolumn{10}{|c|}{ INDICATEURS DE CAPACITES HUMAINES ET NON HUMAINES/ICH } \\
\hline & Y13 & Y14 & Y16 & Y20 & Y24 & Y25 & Y30 & Y39 & Y40 & Y42 \\
\hline PAFPAY03 & $+\mathbf{4 5 , 0 0}$ & 3,69 & 4,03 & 29,48 & 68,71 & $\mathbf{1 , 0 7}$ & $\mathbf{1 1 1 5 4 , 3 2}$ & 4,71 & 22,19 & 5,33 \\
\hline PGMVEY05 & $+3,75$ & 3,38 & 0,39 & 27,04 & 69,63 & 0,09 & $\mathbf{9 5 9 9 , 5 3}$ & 2,14 & 20,35 & 4,20 \\
\hline PATECY12 & $+26,25$ & 3,64 & 2,15 & 29,09 & 64,08 & 0,63 & 12215,29 & 4,29 & 21,8 & 5,76 \\
\hline PEJDCY13 & $+56,25$ & 3,72 & 4,28 & 29,80 & 115,83 & 1,34 & 13151,61 & 5,14 & 22,42 & 6,35 \\
\hline APJY21 & $+60,00$ & 3,01 & 9,63 & 24,06 & 44,74 & 1,43 & 6230,98 & 1,71 & 18,19 & 2,43 \\
\hline NORMES & $+, 0,-$ & 3,75 & 10 & 30 & 50 & 0,75 & 5000 & 1 & 22,75 & 2 \\
\hline
\end{tabular}

Source : Auteur, analyse des données 


\subsubsection{Analyse selon la famille énergétique}

Il ressort que les 5 projets pris en compte ici, sont tous de la famille des énergies «PROMOTIONNELLES, ASCENTIONNELLES ou CONSERVATRICES » comme le montre le tableau ci-dessous.

Pour des projets conçus pour transformer la situation sociale des bénéficiaires, une telle représentativité pourrait ne pas générer d'excellents résultats.

Tableau 6. Matricielle stratégique des profils des projets selon la famille énergétique/Y13

\begin{tabular}{|c|c|c|}
\hline CODES-PROJETS & \multicolumn{2}{|c|}{ FAMILLE ENERGETIQUE / Y13 } \\
\hline PAFPAY03 & $\mathbf{+ 4 5 , 0 0}$ & Promotionnaire , Ascensionnelle ou conservatrice \\
\hline PGMVEY05 & $\mathbf{+ 3 , 7 5}$ & Promotionnaire, Ascensionnelle ou conservatrice \\
\hline PATECY12 & $\mathbf{+ 2 6 , 2 5}$ & Promotionnaire, Ascensionnelle ou conservatrice \\
\hline PEJDCY13 & $\mathbf{+ 5 6 , 2 5}$ & Promotionnaire, Ascensionnelle ou conservatrice \\
\hline APJY21 & $\mathbf{+ 6 0 , 0 0}$ & Promotionnaire, Ascensionnelle ou conservatrice \\
\hline
\end{tabular}

Source : Auteur, analyse des données

\subsubsection{Analyse selon le mobilisateur}

En considérant le mobilisateur, il ressort que tous ces 5 projets ont leur mobilisateur inferieur à la norme indicamétrique $(3,75)$.

Ce qui laisse présager que ces projets ont besoin de voir leur délai d'exécution être rallongé afin qu'ils soient profitables aux bénéficiaires. De facto, tous les moyens financiers, humains et matériels devraient être aussi revus à la hausse, au risque de voir ces projets échoués.

Tableau 7. Matricielle stratégique des profils des projets selon le mobilisateur /Y14

\begin{tabular}{|c|c|}
\hline Code-projets & Mobilisateur / Y14 \\
\hline PAFPAY03 & $\mathbf{3 , 6 9}$ \\
\hline PGMVEY05 & $\mathbf{3 , 3 8}$ \\
\hline PATECY12 & $\mathbf{3 , 6 4}$ \\
\hline PEJDCY13 & $\mathbf{3 , 7 2}$ \\
\hline APJY21 & $\mathbf{3 , 0 1}$ \\
\hline NORMES & $\mathbf{3 , 7 5}$ \\
\hline
\end{tabular}

Source : Auteur, analyse des données

\subsubsection{Analyse selon le régulateur et le stabilisateur}

En ce qui concerne le régulateur, aucun de ces 5 projets n'a une valeur supérieure à la norme (10).

Ce qui laisse présager que ces projets ne sauront faire face à l'hostilité environnementale et n'ont pas un caractère de résilience à la survenue d'éventuelles difficultés. 
Tableau 8. Matricielle stratégique des profils des projets selon le régulateur /Y16

\begin{tabular}{|c|c|c|}
\hline Code-projets & Régulateur / Y16 & Stabilisateur/Y25 \\
\hline PAFPAY03 & $\mathbf{4 , 0 3}$ & $\mathbf{1 , 0 7}$ \\
\hline PGMVEY05 & $\mathbf{0 , 3 9}$ & $\mathbf{0 , 0 9}$ \\
\hline PATECY12 & $\mathbf{2 , 1 5}$ & $\mathbf{0 , 6 3}$ \\
\hline PEJDCY13 & $\mathbf{4 , 2 8}$ & $\mathbf{1 , 3 4}$ \\
\hline APJY21 & $\mathbf{9 , 6 3}$ & $\mathbf{1 , 4 3}$ \\
\hline NORMES & $\mathbf{1 0}$ & $\mathbf{0 , 7 5}$ \\
\hline
\end{tabular}

Source : Auteur, analyse des données

\subsubsection{Analyse selon le taux d'endurance}

Pour ce qui est du taux d'endurance, il ressort que ces 5 projets considérés sont tous déficitaires.

Ces déficits du mobilisateur renseignent sur le fait que ces projets ne seront pas viables. Ce qui nuance leur caractère d'employabilité.

Tableau 9. Matricielle stratégique des profils des projets selon le taux d'endurance /Y20

\begin{tabular}{|c|c|}
\hline Code-projets & Taux d'endurance/ Y20 \\
\hline PAFPAY03 & $\mathbf{2 9 , 4 8}$ \\
\hline PGMVEY05 & $\mathbf{2 7 , 0 4}$ \\
\hline PATECY12 & $\mathbf{2 9 , 0 9}$ \\
\hline PEJDCY13 & $\mathbf{2 9 , 8 0}$ \\
\hline APJY21 & $\mathbf{2 4 , 0 6}$ \\
\hline NORMES & $\mathbf{3 0}$ \\
\hline
\end{tabular}

Source : Auteur, analyse des données

\subsubsection{Analyse selon le taux d'amour humain}

Concernant le taux d'amour humain qui renseigne sur la capacité de ces projets à être profitables aux bénéficiaires, l'on note qu'aucun de ces 5 projets n'a un score supérieur à la norme.

Ces informations voilent le fait que ces projets ne permettront pas à leurs bénéficiaires de pouvoir s'insérer sur le marché du travail ou dans le tissu social, et accéder à leur autonomisation afin de parvenir à l'état de bien-être social.

Tableau 10. Matricielle stratégique des profils des projets selon le taux d'amour humain /Y40

\begin{tabular}{|c|c|}
\hline Code-projets & Taux d'amour humain / Y40 \\
\hline PAFPAY03 & $\mathbf{2 2 , 1 9}$ \\
\hline PGMVEY05 & $\mathbf{2 0 , 3 5}$ \\
\hline PATECY12 & $\mathbf{2 1 , 8}$ \\
\hline PEJDCY13 & $\mathbf{2 2 , 4 2}$ \\
\hline APJY21 & $\mathbf{1 8 , 1 9}$ \\
\hline NORMES & $\mathbf{2 2 . 7 5}$ \\
\hline
\end{tabular}

Source : Auteur, analyse des données 


\subsubsection{Analyse selon le taux de bonne gouvernance}

Il ressort du tableau ci-dessous que tous ces 5 projets sont très exigeants en termes de transparence dans la gestion, voire de saine gestion et surtout en probité morale pour les personnes devant les piloter.

En outre, les scores enregistrés exigent que les gestionnaires de ces différents projets affichent un profil avec un taux de saine gestion et de probité morale égalent ou supérieurs à ceux affichés par lesdits projets.

Si ce n'est pas le cas, les nombreux capitaux mobilisés pour le succès de ces projets seront utilisés à d'autres fins par leurs différents promoteurs ou gestionnaires.

Tableau 11. Matricielle stratégique des profils des projets selon le taux bonne gouvernance /Y39 et le taux d'honnêteté/Y42

\begin{tabular}{|c|c|c|}
\hline Code-projets & $\begin{array}{c}\text { Taux de bonne } \\
\text { gouvernance Y39 }\end{array}$ & $\begin{array}{c}\text { Taux d'honnêteté } \\
\text { Y42 }\end{array}$ \\
\hline PAFPAY03 & $\mathbf{4 , 7 1}$ & $\mathbf{5 , 3 3}$ \\
\hline PGMVEY05 & $\mathbf{2 , 1 4}$ & $\mathbf{4 , 2 0}$ \\
\hline PATECY12 & $\mathbf{4 , 2 9}$ & $\mathbf{5 , 7 6}$ \\
\hline PEJDCY13 & $\mathbf{5 , 1 4}$ & $\mathbf{6 , 3 5}$ \\
\hline APJY21 & $\mathbf{1 , 7 1}$ & $\mathbf{2 , 4 3}$ \\
\hline NORMES & $\mathbf{1}$ & $\mathbf{2}$ \\
\hline
\end{tabular}

Source : Auteur, analyse des données

\subsubsection{Analyse selon le potentiel financier}

L'analyse du potentiel financier révèle que tous ces 5 projets ont un potentiel financier largement supérieur à la norme (5000). Ceci laisse entrevoir que ces projets seront budgétivores. Ce qui exigerait une transparence dans la gestion des fonds affectés à ces projets et bonne probité morale des promoteurs, si l'on veut s'inscrire dans une logique d'efficacité et d'efficience.

Tableau 12. Matricielle stratégique des profils des projets selon potentiel financier/Y30

\begin{tabular}{|c|c|}
\hline Code-projets & Potentiel financier Y30 \\
\hline PAFPAY03 & $\mathbf{1 1 1 5 4 , 3 2}$ \\
\hline PGMVEY05 & $\mathbf{9 5 9 9 , 5 3}$ \\
\hline PATECY12 & $\mathbf{1 2 2 1 5 , 2 9}$ \\
\hline PEJDCY13 & $\mathbf{1 3 1 5 1 , 6 1}$ \\
\hline APJY21 & $\mathbf{6 2 3 0 , 9 8}$ \\
\hline NORMES & $\mathbf{5 0 0 0}$ \\
\hline
\end{tabular}

Source : Auteur, analyse des données

\subsection{Analyse des projets à fort taux de faisabilité}

Le tableau ci-dessous présente les caractéristiques des projets qui se démarquent par leur taux de faisabilité acceptable. 
Tableau 13. Matricielle stratégique des 8 projets réalisables

\begin{tabular}{|c|c|c|c|c|c|c|c|c|c|c|}
\hline \multirow{2}{*}{ CODES-PROJETS } & \multicolumn{8}{|c|}{ INDICATEURS DE CAPACITES HUMAINES ET NON HUMAINES/ICH } \\
\cline { 2 - 13 }$y$ & Y13 & Y14 & Y16 & Y20 & Y24 & Y25 & Y30 & Y39 & Y40 & Y42 \\
\hline PFA/AJDY06 & $\mathbf{- 1 3 8 , 7 5}$ & $\mathbf{3 , 8 8}$ & $\mathbf{6 , 5 7}$ & $\mathbf{3 1 , 0 8}$ & $\mathbf{7 2} \mathbf{2 2 9}$ & $\mathbf{3 , 3 0}$ & $\mathbf{2 6 1 2 2 , 3 2}$ & $\mathbf{8 , 1 4}$ & $\mathbf{2 3 , 3 9}$ & $\mathbf{1 0 , 6 3}$ \\
\hline P2APHFCY9 & $\mathbf{- 4 8 , 7 5}$ & $\mathbf{4 , 0 0}$ & $\mathbf{2 , 8 8}$ & $\mathbf{3 2 , 0 4}$ & $\mathbf{7 8 , 2 7}$ & $\mathbf{1 , 1 6}$ & $\mathbf{2 1 9 1 1 , 9 4}$ & $\mathbf{8 , 1 4}$ & $\mathbf{2 4 , 1 1}$ & $\mathbf{8 , 7 8}$ \\
\hline P2AY10 & $\mathbf{+ 4 1 , 2 5}$ & $\mathbf{4 , 1 7}$ & $\mathbf{5 , 8 1}$ & $\mathbf{3 3 , 3 4}$ & $\mathbf{1 3 0 , 6 0}$ & $\mathbf{0 , 9 8}$ & $\mathbf{7 1 0 4 , 2 4}$ & $\mathbf{3 , 0 0}$ & $\mathbf{2 5 , 0 9}$ & $\mathbf{3 , 8 4}$ \\
\hline FSETHIMOY11 & $\mathbf{+ 4 8 , 7 5}$ & $\mathbf{3 , 8 5}$ & $\mathbf{2 , 4 0}$ & $\mathbf{3 0 , 7 8}$ & $\mathbf{6 3 , 7 4}$ & $\mathbf{1 , 1 6}$ & $\mathbf{2 0 3 4 6 , 6 7}$ & $\mathbf{7 , 2 9}$ & $\mathbf{2 3 , 1 6}$ & $\mathbf{1 0 , 1 4}$ \\
\hline PSRMAEFY14 & $\mathbf{+ 6 7 , 5 0}$ & $\mathbf{3 , 8 4}$ & $\mathbf{4 , 3 9}$ & $\mathbf{3 0 , 7 4}$ & $\mathbf{5 1 , 4 6}$ & $\mathbf{1 , 6 1}$ & $\mathbf{1 5 3 7 2 , 3 8}$ & $\mathbf{7 , 7 1}$ & $\mathbf{2 3 , 1 3}$ & $\mathbf{7 , 8 5}$ \\
\hline SCAEDY17 & $\mathbf{+ 7 5 , 0 0}$ & $\mathbf{3 , 8 5}$ & $\mathbf{6 , 4 6}$ & $\mathbf{3 0 , 8 3}$ & $\mathbf{9 1 , 1 5}$ & $\mathbf{1 , 7 9}$ & $\mathbf{1 1 6 1 4 , 4 6}$ & $\mathbf{3 , 4 3}$ & $\mathbf{2 3 , 1 8}$ & $\mathbf{5 , 8 0}$ \\
\hline P2AEIJY19 & $\mathbf{+ 5 6 , 2 5}$ & $\mathbf{3 , 8 2}$ & $\mathbf{3 , 5 6}$ & $\mathbf{3 0 , 5 8}$ & $\mathbf{6 1 , 6 4}$ & $\mathbf{1 , 3 4}$ & $\mathbf{1 5 9 4 4 , 9 9}$ & $\mathbf{8 , 1 4}$ & $\mathbf{2 3 , 0 1}$ & $\mathbf{7 , 9 0}$ \\
\hline C2DEY20 & $\mathbf{+ 6 0}$ & $\mathbf{3 , 8 2}$ & $\mathbf{4 , 4 5}$ & $\mathbf{3 0 , 5 6}$ & $\mathbf{1 5 6 , 1 3}$ & $\mathbf{1 , 4 3}$ & $\mathbf{1 3 4 8 3 , 6 5}$ & $\mathbf{6 , 8 6}$ & $\mathbf{2 2 , 9 9}$ & $\mathbf{6 , 6 7}$ \\
\hline NORMES & $+, 0,-$ & $\mathbf{3 , 7 5}$ & $\mathbf{1 0}$ & $\mathbf{3 0}$ & $\mathbf{5 0}$ & $\mathbf{0 , 7 5}$ & $\mathbf{5 0 0 0}$ & $\mathbf{1}$ & $\mathbf{2 2 , 7 5}$ & $\mathbf{2}$ \\
\hline
\end{tabular}

\subsubsection{Analyse selon la famille énergétique}

En considérant la famille énergétique, Il ressort que sur les 8 projets considérés, 2 sont de la famille des énergies «CREATRICES, TRANSFORMATRICES » et 6 sont de la famille des énergies «PROMOTIONNELLES, ASCENTIONNELLES ou CONSERVATRICES » et 0 projet issu de la famille des énergies CENTRALISATRICES, comme le montre le tableau ci-dessous.

Tableau 14. Matricielle stratégique des profils des projets selon la famille énergétique/Y13

\begin{tabular}{|c|c|}
\hline CODES-PROJETS & FAMILLE ENERGETIQUE / Y13 \\
\hline PFA/AJDY06 & $\mathbf{- 1 3 8 , 7 5}$ \\
\hline P2APHFCY9 & $\mathbf{- 4 8 , 7 5}$ \\
\hline P2AY10 & $\mathbf{+ 4 1 , 2 5}$ \\
\hline FSETHIMOY11 & $\mathbf{+ 4 8 , 7 5}$ \\
\hline PSRMAEFY14 & $\mathbf{+ 6 7 , 5 0}$ \\
\hline SCAEDY17 & $\mathbf{+ 7 5 , 0 0}$ \\
\hline P2AEIJY19 & $\mathbf{+ 5 6 , 2 5}$ \\
\hline C2DEY20 & $\mathbf{+ 6 0}$ \\
\hline
\end{tabular}

Source : Auteur, analyse des données 


\subsubsection{Analyse selon le mobilisateur}

Les valeurs du mobilisateur des 8 projets considérés révèlent que celles-ci sont supérieures à la norme indicamétrique $(3,75)$. Ce qui présage que ces projets mettront peu de temps pour donner satisfaction. Aussi, les moyens humains, financiers et matériels mobilises pendant la conception pourront tenir dans les délais impartis. Ceci constitue un réel atout, gage de succès.

Tableau 15. Matricielle stratégique des profils des projets selon le mobilisateur /Y14

\begin{tabular}{|c|c|}
\hline Code-projets & Mobilisateur / Y14 \\
\hline PFA/AJDY06 & $\mathbf{3 , 8 8}$ \\
\hline P2APHFCY9 & $\mathbf{4 , 0 0}$ \\
\hline P2AY10 & $\mathbf{4 , 1 7}$ \\
\hline FSETHIMOY11 & $\mathbf{3 , 8 5}$ \\
\hline PSRMAEFY14 & $\mathbf{3 , 8 4}$ \\
\hline SCAEDY17 & $\mathbf{3 , 8 5}$ \\
\hline P2AEIJY19 & $\mathbf{3 , 8 2}$ \\
\hline C2DEY20 & $\mathbf{3 , 8 2}$ \\
\hline NORMES & $\mathbf{3 , 7 5}$ \\
\hline
\end{tabular}

Source : Auteur, analyse des données

\subsubsection{Analyse selon le régulateur et le stabilisateur}

Il ressort du tableau ci-dessous que la totalité des 8 projets considérés affichent des valeurs déficitaires par rapport à la norme indicamétrique (10). Cependant, la totalité de ces projets enregistrent des valeurs du stabilisateur supérieures à la norme $(0,75)$. En outre, pour pallier au déficit du régulateur, les indicateurs Y14 (mobilisateur) et Y20 (taux d'endurance) combinés au bon stabilisateur devraient être performants afin de servir de relais et permettre à tous ces projets de pouvoir tenir face à l'hostilité de l'environnement et être résilients en cas difficultés majeures.

Tableau 16. Matricielle stratégique des profils des projets selon le régulateur /Y16 et le stabilisateur/Y25

\begin{tabular}{|c|c|c|}
\hline Code-projets & Régulateur / Y16 & Stabilisateur/ Y25 \\
\hline PFA/AJDY06 & $\mathbf{6 , 5 7}$ & $\mathbf{3 , 3 0}$ \\
\hline P2APHFCY9 & $\mathbf{2 , 8 8}$ & $\mathbf{1 , 1 6}$ \\
\hline P2AY10 & $\mathbf{5 , 8 1}$ & $\mathbf{0 , 9 8}$ \\
\hline FSETHIMOY11 & $\mathbf{2 , 4 0}$ & $\mathbf{1 , 1 6}$ \\
\hline PSRMAEFY14 & $\mathbf{4 , 3 9}$ & $\mathbf{1 , 6 1}$ \\
\hline SCAEDY17 & $\mathbf{6 , 4 6}$ & $\mathbf{1 , 7 9}$ \\
\hline P2AEIJY19 & $\mathbf{3 , 5 6}$ & $\mathbf{1 , 3 4}$ \\
\hline C2DEY20 & $\mathbf{4 , 4 5}$ & $\mathbf{1 , 4 3}$ \\
\hline NORMES & $\mathbf{1 0}$ & $\mathbf{0 , 7 5}$ \\
\hline
\end{tabular}

Source : Auteur, analyse des données 


\subsubsection{Analyse selon le taux d'endurance}

Pour ce qui est du taux d'endurance, tous les 8 projets sont excédentaires par rapport à la norme (30).

Cette information laisse entrevoir que tous ces projets, permettront en termes de mobilisation et d'endurance de relayer leurs déficits au niveau du régulateur, ce qui de facto garanti leur faisabilité et leur efficacité.

Tableau 17. Matricielle stratégique des profils des projets selon le taux d'endurance /Y20

\begin{tabular}{|c|c|}
\hline Code-projets & Taux d'endurance/ Y20 \\
\hline PFA/AJDY06 & $\mathbf{3 1 , 0 8}$ \\
\hline P2APHFCY9 & $\mathbf{3 2 , 0 4}$ \\
\hline P2AY10 & $\mathbf{3 3 , 3 4}$ \\
\hline FSETHIMOY11 & $\mathbf{3 0 , 7 8}$ \\
\hline PSRMAEFY14 & $\mathbf{3 0 , 7 4}$ \\
\hline SCAEDY17 & $\mathbf{3 0 , 8 3}$ \\
\hline P2AEIJY19 & $\mathbf{3 0 , 5 8}$ \\
\hline C2DEY20 & $\mathbf{3 0 , 5 6}$ \\
\hline NORMES & $\mathbf{3 0}$ \\
\hline
\end{tabular}

Source : Auteur, analyse des données

\subsubsection{Analyse selon taux d'amour humain}

En considérant le degré d'amour humain, l'on note que l'ensemble des 8 projets sont excédentaires en se référant à la norme indicamétrique $(22,75)$.

Ceci renseigne sur le fait que ces projets ont un caractère humain. Ils pourront dans leur phase de réalisation parvenir à assurer l'insertion sur le marché du travail et dans le tissu social ainsi que l'autonomisation de leurs bénéficiaires.

Tableau 18. Matricielle stratégique des profils des projets selon le taux d'amour humain /Y40

\begin{tabular}{|c|c|}
\hline Code-projets & Mobilisateur / Y40 \\
\hline PFA/AJDY06 & $\mathbf{2 3 , 3 9}$ \\
\hline P2APHFCY9 & $\mathbf{2 4 , 1 1}$ \\
\hline P2AY10 & $\mathbf{2 5 , 0 9}$ \\
\hline FSETHIMOY11 & $\mathbf{2 3 , 1 6}$ \\
\hline PSRMAEFY14 & $\mathbf{2 3 , 1 3}$ \\
\hline CAFPCDY16 & $\mathbf{2 3 , 1 8}$ \\
\hline SCAEDY17 & $\mathbf{2 3 , 0 1}$ \\
\hline PRS2ECY18 & $\mathbf{2 2 , 9 9}$ \\
\hline NORMES & $\mathbf{2 2 , 7 5}$ \\
\hline
\end{tabular}

Source : Auteur, analyse des données

\subsubsection{Analyse selon le taux de bonne gouvernance et le taux d'honnêteté}

Pour ce qui est des exigences en matière de saine gestion et de bonne gouvernance, tous ces 8 projets requièrent un niveau élevé en termes de transparence et de probité morale. Ce qui implique la prise en compte des 
critères d'intégrité et de capacité de saine gestion dans le choix des gestionnaires ou des promoteurs desdits projets.

Tableau 19. Matricielle stratégique des profils des projets selon le taux bonne gouvernance /Y39 et le taux d'honnêteté/Y42

\begin{tabular}{|c|c|c|}
\hline Code-projets & Y39 & Y42 \\
\hline PFA/AJDY06 & $\mathbf{8 , 1 4}$ & $\mathbf{1 0 , 6 3}$ \\
\hline P2APHFCY9 & $\mathbf{8 , 1 4}$ & $\mathbf{8 , 7 8}$ \\
\hline P2AY10 & $\mathbf{3 , 0 0}$ & $\mathbf{3 , 8 4}$ \\
\hline FSETHIMOY11 & $\mathbf{7 , 2 9}$ & $\mathbf{1 0 , 1 4}$ \\
\hline PSRMAEFY14 & $\mathbf{7 , 7 1}$ & $\mathbf{7 , 8 5}$ \\
\hline SCAEDY17 & $\mathbf{3 , 4 3}$ & $\mathbf{5 , 8 0}$ \\
\hline P2AEIJY19 & $\mathbf{8 , 1 4}$ & $\mathbf{7 , 9 0}$ \\
\hline C2DEY20 & $\mathbf{6 , 8 6}$ & $\mathbf{6 , 6 7}$ \\
\hline NORMES & $\mathbf{1}$ & $\mathbf{2}$ \\
\hline
\end{tabular}

Source : Auteur, analyse des données

\subsubsection{Analyse selon le potentiel financier}

Les scores excédentaires affichés par ces 8 projets au niveau des ressources financières laissent entrevoir que ces initiatives nécessiteront de lourds investissements, ce qui nécessitent que les fonds injectés soient gérés avec beaucoup de prudence, de transparence et de façon efficiente.

Tableau 20. Matricielle stratégique des profils des projets selon potentiel financier/Y30

\begin{tabular}{|c|c|}
\hline Code-projets & Y30 \\
\hline PFA/AJDY06 & $\mathbf{2 6 1 2 2 , 3 2}$ \\
\hline P2APHFCY9 & $\mathbf{2 1 9 1 1 , 9 4}$ \\
\hline P2AY10 & $\mathbf{7 1 0 4 , 2 4}$ \\
\hline FSETHIMOY11 & $\mathbf{2 0 3 4 6 , 6 7}$ \\
\hline PSRMAEFY14 & $\mathbf{1 5 3 7 2 , 3 8}$ \\
\hline SCAEDY17 & $\mathbf{1 1 6 1 4 , 4 6}$ \\
\hline P2AEIJY19 & $\mathbf{1 5 9 4 4 , 9 9}$ \\
\hline C2DEY20 & $\mathbf{1 3 4 8 3 , 6 5}$ \\
\hline NORMES & $\mathbf{3 0}$ \\
\hline
\end{tabular}

Source : Auteur, analyse des données

\section{Synthèse des caractéristiques des projets et programmes de l'étude}

En supposant que tout projet satisfaisant au moins 7 indicateurs à valeurs supérieures aux normes respectives indicamétriques, notre étude révèlent que 8 des 13 projets inclus dans notre échantillon, affichaient à la phase de conception, de forte probabilité de succès, de façon efficiente.

Quant aux autres projets, qui n'enregistrent de valeurs satisfaisantes des indicateurs par rapport à la norme indicamétrique, leur succès reste conditionné à d'énormes précautions dans l'allocation des ressources humaines, matérielles et financières. 
Ces résultats mettent en exergue la pertinence de l'outil indicamétrique dans la phase de conception des projets et programmes. Toute chose peu discernable par les outils classiques d'analyse de risques de projets.

Tableau 21. Recaitulatif des Atouts et Faiblesses des Divers Projets

\begin{tabular}{|c|c|c|c|}
\hline \multirow{2}{*}{ CODES-PROJETS } & \multicolumn{2}{|c|}{$\begin{array}{c}\text { OBSERVATIONS SUR LES } \\
\text { 9 INDICATEURS }\end{array}$} & \multirow{2}{*}{$\begin{array}{c}\text { VERDICTS } \\
\text { (Probablement) }\end{array}$} \\
\cline { 2 - 3 } & ATOUTS & FAIBLESSES & \\
\hline PAFPAY03 & 5 & 4 & ECHEC \\
\hline PGMVEY05 & 4 & 5 & ECHEC \\
\hline PFA/AJDY06 & 8 & 1 & SUCCES \\
\hline P2APHFCY9 & 8 & 1 & SUCCES \\
\hline P2AY10 & 8 & 1 & SUCCES \\
\hline FSETHIMOY11 & 8 & 1 & SUCCES \\
\hline PATECY12 & 4 & 5 & ECHEC \\
\hline PEJDCY13 & 5 & 4 & ECHEC \\
\hline PSRMAEFY14 & 8 & 1 & SUCCES \\
\hline SCAEDY17 & 8 & 1 & SUCCES \\
\hline P2AEIJY19 & 8 & 1 & SUCCES \\
\hline C2DEY20 & 8 & 1 & SUCCES \\
\hline APJY21 & 4 & 5 & ECHEC \\
\hline
\end{tabular}

Source : Auteur, analyse des données

\section{Conclusions, limites et implications}

Cette étude nous a permis de mettre en exergue les facteurs subjectifs occultés par les outils classiques d'analyse et de gestion de projets, basés sur des indicateurs objectifs. En outre, l'analyse des projets par la méthode indicamétrique s'avère nécessaire, voire indispensable, dans un contexte de rareté des ressources, qui impose l'fficience et l'efficacité pour toute action.

\subsection{Principales conclusions de l'étude}

D'une manière générale, cette étude visait à contribuer à l'amélioration de l'efficacité et l'efficience des projets et programmes, notamment dans le secteur emploi-formation.

De façon spécifique, il s'agissait de :

- Identifier les facteurs subjectifs de risques liés à la conception, la gestion et l'impact des projets et programmes ;

- Proposer des indicateurs subjectifs et mesurables pour l'analyse des projets et programmes ;

- Identifier les facteurs déterminants de succès des projets et programmes ;

- Identifier les caractéristiques intrinsèques individuelles des promoteurs et gestionnaires de projets et programmes. 
$\mathrm{Au}$ terme de notre recherche marquée par une brève revue de la littérature sur l'indicamétrie, la présentation des aspects méthodologiques et la définition des différents concepts en lien avec la gestion des projets, nous avons abouti aux principales conclusions suivantes :

De façon globale, il ressort que la science indicamétrique par sa méthodologie originale et novatrice, permet d'identifier les caractéristiques intrinsèques individuelles des personnes physiques et morales impliquées dans la conception et la gestion des projets et programmes, ainsi que les caractéristiques de l'environnement interne et externe, en vue d'atteindre l'efficacité et l'efficience.

Plus précisément, il est à noter que :

* L'analyse indicamétrique permet de déceler les facteurs de succès et d'échec en amont, dès la phase de conception des projets, accroit l'efficience et l'efficacité des résultats attendus ;

* La prise en compte des caractéristiques de l'environnement interne et externe des projets accroit leur probabilité de succès ;

* Le choix de la dénomination de tout projet ou programme influence son mode de gestion ;

La prise en compte des caractéristiques et des capacités intrinsèques des promoteurs ou gestionnaires des projets dans leur mise en œuvre accroit significativement leur probabilité de succès.

\subsection{Limites et implications de l'étude}

La principale limite de notre étude réside dans le fait que le manque de données sur les gestionnaires des projets de notre l'étude, ne nous a pas permis d'inclure les caractéristiques de ceux-ci dans nos analyses. Notre travail pourrait donc être amélioré à travers des analyse plus affinées intégrant ces paramètres.

\subsection{Recommandations et propositions d'actions}

Au regard des principales conclusions de l'étude nous formulons les recommandations suivantes :

\section{A l'endroit de la communauté des chercheurs}

* Intégrer la science indicamétrique dans l'analyse de la structure du marché du travail ;

Développer des méthodes d'analyse et des indicateurs subjectifs indicamétriques dans la conception des outils de gestion des projets et programmes ; 


\section{A l'endroit du Gouvernement,}

* Promouvoir l'emploi des jeunes, à travers la redynamisation des actions des structures en charge de leur insertion et leur employabilité ; Mettre l'accent sur l'industrialisation, notamment la transformation des produits agricoles au niveau local, en vue d'accroitre les opportunités d'emploi ;

* Promouvoir l'emploi des femmes, notamment celles diplômées de l'enseignement technique et professionnel et de l'enseignement supérieur, à travers la création de structures spécifiques dédiées à la promotion de l'emploi féminin ;

* Actualiser les curricula de formation des structures de formation, en vue de les adapter à la structure de l'économie nationale ;

* Développer la culture de l'entrepreneuriat, notamment chez les jeunes et les femmes, ainsi que les enfants dès l'école primaire ;

* Poursuivre et accentuer les efforts de formalisation des activités informelles par la facilitation de création d'entreprises, notamment de PME/PMI nationales ;

* Analyser les capacités intrinsèques des porteurs de projets avant l'octroi ou la validation du financement de tout projet.

\section{References:}

1. Affi, M. L. (2007). in L'indicamétrie et ses instruments, Abidjan, CIDI-Production.

2. Deazon, A. (2009). De la Ligne Droite à la Spirale, Abidjan, COMIPA, Production.

3. Diabaté, M. (1969). Du sous-développement au blocage du développement, Edition Présence Africaine, Paris.

4. Diabaté, M. (1974). Le modèle ivoirien de développement, Thèse de Doctorat d'Etat, Paris, Sorbonne.

5. Diabaté, M. (1999). Théories et pratiques du développement et de l'entreprenariat (de l'approche classique à l'approche Indicamétrique) Université de Bouaké, Indicame Edition.

6. Diabaté, M. (2007). Matricielle stratégique des performances capacitaire profils multi capacitaires comparés de 0Y87.75 et 0Y67.50. CIDI Production, Paris.

7. Diabaté, M. (2009). La Révolution Indicamétrique ou le Réveil d'un continent pour le salut de l'humanité et du Monde, Abidjan, COMIPA Production.

8. Dieudonné, J. (1962). Les Méthodes Axiomatiques Moderne et les Fondements des Mathématiques, in Les Grands Courants de la Pensée Mathématique, Paris, Blanchard. 
9. Drouin, P. (1980). journal Le Monde-Edition Internationale du 31 Janvier 1980.

10. Encyclopédie Bordas, volume 36 Edition 1970, des Anthroposociologues de l'époque coloniale comme Marcel Mauss et LéviStrauss en parlaient dans leurs travaux en Asie et à Polynésie.

11. Giuseppe, L. \& Tendero, P. E. (2008). l'alphabet, la machine et l'ADN/in évolution des concepts fondamentaux de la biologie du XXI siècle (Miquel éd.) DE BOECK Paris.

12. Prof. Fournier (1963). Cours d'économie de la médecine, IEDES.

13. Prof. Kouakou C. (2019). améliorer les politiques d'emploi jeune en Afrique francophone: cas de la Cote d'Ivoire, CRDI, CREMIDE, Abidjan, Côte d'Ivoire.

14. Serge O. (2018). Science de l'indicamétrie, Cours Chrono profil : activités et familles énergétiques, Abidjan, Côte d'ivoire.

15. Wittgenstein, L. (1961). Tractatus Logico-philosophicus, Paris, Trad. Pierre Klossowski, Gallimard.

16. http://fr.wikipedia.org

17. www.info.univ-angers.fr

18. https://www.agenceemploijeunes.ci/ 


\section{ANNEXE}

\section{LES CARTES CAPACITAIRES DES PROJETS}

\section{Projet d'Appui à la Formation de la Population Active (PAFPA)}

\begin{tabular}{|c|c|r|r|r|r|r|r|r|r|r|r|r|}
\hline ICH & Y13 & Y14 & Y15 & Y16 & Y6 & Y19 & Y20 & Y22 & Y24 & Y25 & Y26 & Y28 \\
\hline $\begin{array}{c}\text { PAFPAY } \\
03\end{array}$ & $+45,00$ & 3,69 & 0,94 & 4,03 & 261,37 & 95,55 & 29,48 & 2,55 & 68,71 & 1,07 & 1,25 & 1,75 \\
\hline Norme & $\begin{array}{c}+, 0,- \\
\text { Yn }\end{array}$ & 3,75 & 0,75 & 10 & 100 & 100 & 30 & 1 & 50 & 0,75 & 0,5 & 1 \\
\hline
\end{tabular}

\begin{tabular}{|c|c|c|c|r|r|r|r|}
\hline Y30 & Y39 & Y40 & Y41 & \multicolumn{1}{|c|}{ Y42 } & \multicolumn{1}{|c|}{ Y43 } & \multicolumn{1}{c|}{ Y44 } & \multicolumn{1}{|c|}{ Y45 } \\
\hline 11154,32 & 4,71 & 22,19 & 3,58 & 5,33 & 4,66 & 13,82 & 49,25 \\
\hline 5000 & 1 & 22,75 & 3,75 & 2 & 1,5 & 15 & 50 \\
\hline
\end{tabular}

2. Le projet «Gérez Mieux votre Entreprise » (GERME)

\begin{tabular}{|c|c|c|c|c|r|r|r|r|r|r|r|r|}
\hline ICH & Y13 & Y14 & Y15 & Y16 & Y6 & Y19 & Y20 & Y22 & Y24 & Y25 & Y26 & Y28 \\
\hline $\begin{array}{c}\text { PGMVE } \\
\text { Y05 }\end{array}$ & $+3,75$ & 3,38 & 0,99 & 0,39 & 185,10 & 95,50 & 27,04 & 1,58 & 69,63 & 0,09 & 1,58 & 3,25 \\
\hline Norme & $\begin{array}{c}+, 0,- \\
\text { Yn }\end{array}$ & 3,75 & 0,75 & 10 & 100 & 100 & 30 & 1 & 50 & 0,75 & 0,5 & 1 \\
\hline
\end{tabular}

\begin{tabular}{|c|c|c|r|r|r|r|r|}
\hline Y30 & Y39 & Y40 & \multicolumn{1}{c|}{ Y41 } & \multicolumn{1}{c|}{ Y42 } & \multicolumn{1}{c|}{ Y43 } & \multicolumn{1}{c|}{ Y44 } & \multicolumn{1}{c|}{ Y45 } \\
\hline 9599,53 & 2,14 & 20,35 & 3,58 & 4,20 & 3,68 & 12,67 & 50,55 \\
\hline 5000 & 1 & 22,75 & 3,75 & 2 & 1,5 & 15 & 50 \\
\hline
\end{tabular}

3. Le Projet de Formation par Apprentissage (PFA) dans le cadre du Programme d'Absorption des Jeunes Déscolarisés (PAJD)

\begin{tabular}{|c|c|c|c|c|c|r|r|r|r|r|r|r|}
\hline ICH & Y13 & Y14 & Y15 & Y16 & Y6 & Y19 & Y20 & Y22 & Y24 & Y25 & Y26 & Y28 \\
\hline $\begin{array}{c}\text { PFA/AJ } \\
\text { DY06 }\end{array}$ & $-138,75$ & 3,88 & 1,10 & 6,57 & 624,43 & 95,55 & 31,08 & 2,55 & 72,29 & 3,30 & 1,25 & 2,55 \\
\hline Norme & $+, 0,-$ Yn & 3,75 & 0,75 & 10 & 100 & 100 & 30 & 1 & 50 & 0,75 & 0,5 & 1 \\
\hline
\end{tabular}




\begin{tabular}{|r|r|r|r|r|r|r|r|}
\hline Y30 & Y39 & Y40 & Y41 & \multicolumn{1}{c|}{ Y42 } & \multicolumn{1}{c|}{ Y43 } & \multicolumn{1}{c|}{ Y44 } & \multicolumn{1}{c|}{ Y45 } \\
\hline $\begin{array}{r}26122,3 \\
2\end{array}$ & 8,14 & 23,39 & 3,25 & 10,63 & 9,30 & 14,57 & 51,25 \\
\hline 5000 & 1 & 22,75 & 3,75 & 2 & 1,5 & 15 & 50 \\
\hline
\end{tabular}

4. Le Projet d'Appui et d'Accès des Personnes Handicapées à la Formation Professionnelle (PAAPHFP)

\begin{tabular}{|c|c|c|c|c|r|r|r|r|r|r|r|r|}
\hline ICH & Y13 & Y14 & Y15 & Y16 & Y6 & Y19 & Y20 & Y22 & Y24 & Y25 & Y26 & Y28 \\
\hline $\begin{array}{c}\text { P2APHF } \\
\text { CY9 }\end{array}$ & $-48,75$ & 4,00 & 1,04 & 2,88 & 532,90 & 95,50 & 32,04 & 2,55 & 78,27 & 1,16 & 1,25 & 2,36 \\
\hline Norme & $\begin{array}{c}+, 0,- \\
\text { Yn }\end{array}$ & 3,75 & 0,75 & 10 & 100 & 100 & 30 & 1 & 50 & 0,75 & 0,5 & 1 \\
\hline
\end{tabular}

\begin{tabular}{|c|c|c|r|r|r|r|r|}
\hline Y30 & Y39 & Y40 & Y41 & Y42 & Y43 & \multicolumn{1}{c|}{ Y44 } & \multicolumn{1}{c|}{ Y45 } \\
\hline 21911,94 & 8,14 & 24,11 & 3,55 & 8,78 & 7,68 & 15,02 & 48,88 \\
& & & & & & & \\
\hline 5000 & 1 & 22,75 & 3,75 & 2 & 1,5 & 15 & 50 \\
\hline
\end{tabular}

\section{Le Programme d'Aide à l'Embauche (PAE)}

\begin{tabular}{|c|c|c|c|c|r|r|r|r|r|r|r|r|}
\hline ICH & Y13 & Y14 & Y15 & Y16 & Y6 & Y19 & Y20 & Y22 & Y24 & Y25 & Y26 & Y28 \\
\hline $\begin{array}{c}\text { P2AY1 } \\
0\end{array}$ & $+41,25$ & 4,77 & 0,93 & 5,81 & $\begin{array}{r}281,1 \\
8\end{array}$ & 98,25 & 33,34 & 2,55 & 130,60 & 0,98 & 1,25 & 2,66 \\
\hline Norme & $\begin{array}{c}+, 0,- \\
\text { Yn }\end{array}$ & 3,75 & 0,75 & 10 & 100 & 100 & 30 & 1 & 50 & 0,75 & 0,5 & 1 \\
\hline
\end{tabular}

\begin{tabular}{|c|c|c|c|r|r|r|r|}
\hline Y30 & Y39 & Y40 & Y41 & Y42 & Y43 & \multicolumn{1}{c|}{ Y44 } & \multicolumn{1}{c|}{ Y45 } \\
\hline 71042,24 & 3,00 & 25,09 & 3,58 & 3,84 & 3,36 & 15,63 & 45,95 \\
\hline 5000 & 1 & 22,75 & 3,75 & 2 & 1,5 & 15 & 50 \\
\hline
\end{tabular}




\section{Le Fonds de soutien à l'emploi par les travaux d'utilité publique à haute intensité de main d'ouvre (FSE-THIMO)}

\begin{tabular}{|c|c|r|r|r|r|r|r|r|r|r|r|r|}
\hline ICH & Y13 & Y14 & Y15 & Y16 & Y6 & Y19 & Y20 & Y22 & Y24 & Y25 & Y26 & Y28 \\
\hline $\begin{array}{c}\text { FSETHIM } \\
\text { OY11 }\end{array}$ & $+48,75$ & 3,85 & 0,96 & 2,40 & 578,44 & 90,25 & 30,78 & 2,58 & 63,74 & 1,16 & 1,58 & 3,56 \\
\hline Norme & $\begin{array}{c}+, 0,- \\
\text { Yn }\end{array}$ & 3,75 & 0,75 & 10 & 100 & 100 & 30 & 1 & 50 & 0,75 & 0,5 & 1 \\
\hline
\end{tabular}

\begin{tabular}{|r|r|r|r|r|r|r|r|}
\hline Y30 & Y39 & Y40 & Y41 & \multicolumn{1}{c|}{ Y42 } & \multicolumn{1}{c|}{ Y43 } & \multicolumn{1}{c|}{ Y44 } & \multicolumn{1}{c|}{ Y45 } \\
\hline 20346,6 & 7,29 & 23,16 & 3,58 & 10,14 & 8,88 & 14,43 & 45,88 \\
7 & & & & & & & \\
\hline 5000 & 1 & 22,75 & 3,75 & 2 & 1,5 & 15 & 50 \\
\hline
\end{tabular}

\section{Le Projet d'Appui au Traitement Economique du Chômage (PATEC)}

\begin{tabular}{|c|c|c|c|c|c|c|c|c|c|c|c|c|}
\hline ICH & Y13 & Y14 & Y15 & Y16 & Y6 & Y19 & Y20 & Y22 & Y24 & Y25 & Y26 & Y28 \\
\hline $\begin{array}{c}\text { PATEC } \\
\text { Y12 }\end{array}$ & $+26,25$ & 3,64 & 0,97 & 2,15 & 296,89 & 98,85 & 29,09 & 2,36 & 64,08 & 0,63 & 1,25 & 2,56 \\
\hline Norme & $\begin{array}{c}+, 0,- \\
\text { Yn }\end{array}$ & 3,75 & 0,75 & 10 & 100 & 100 & 30 & 1 & 50 & 0,75 & 0,5 & 1 \\
\hline
\end{tabular}

\begin{tabular}{|c|c|c|c|c|c|c|c|}
\hline Y30 & Y39 & Y40 & Y41 & Y42 & Y43 & \multicolumn{1}{|c|}{ Y44 } & \multicolumn{1}{c|}{ Y45 } \\
\hline 12215,29 & 4,29 & 21,89 & 3,15 & 5,76 & 5,04 & 13,64 & 49,50 \\
& & & & & & & \\
\hline 5000 & 1 & 22,75 & 3,75 & 2 & 1,5 & 15 & 50 \\
\hline
\end{tabular}

8. Projet Emploi des Jeunes et Développement des Compétences (PEJEDEC)

\begin{tabular}{|c|c|c|c|c|c|c|c|c|c|c|c|c|}
\hline ICH & Y13 & Y14 & Y15 & Y16 & Y6 & Y19 & Y20 & Y22 & Y24 & Y25 & Y26 & Y28 \\
\hline $\begin{array}{c}\text { PEJDC } \\
\text { Y13 }\end{array}$ & $+56,25$ & 3,72 & 0,94 & 4,28 & 342,88 & 98,75 & 29,80 & 1,25 & 115,83 & 1,34 & 1,25 & 2,22 \\
\hline Norme & $\begin{array}{c}+, 0,- \\
\text { Yn }\end{array}$ & 3,75 & 0,75 & 10 & 100 & 100 & 30 & 1 & 50 & 0,75 & 0,5 & 1 \\
\hline
\end{tabular}

\begin{tabular}{|c|c|c|c|r|r|r|r|}
\hline Y30 & Y39 & Y40 & Y41 & Y42 & Y43 & Y44 & \multicolumn{1}{|c|}{ Y45 } \\
\hline 13151,61 & 5,14 & 22,42 & 3,58 & 6,35 & 5,55 & 13,97 & 45,85 \\
\hline 5000 & 1 & 22,75 & 3,75 & 2 & 1,5 & 15 & 50 \\
\hline
\end{tabular}




\section{Programme spécial de requalification et de mise en adéquation emploi-formation (PSRMAEF)}

\begin{tabular}{|c|c|c|c|c|c|c|c|c|c|c|c|c|}
\hline ICH & Y13 & Y14 & Y15 & Y16 & Y6 & Y19 & Y20 & Y22 & Y24 & Y25 & Y26 & Y28 \\
\hline $\begin{array}{c}\text { PSRMA } \\
\text { EFY14 }\end{array}$ & $+67,50$ & 3,84 & 0,94 & 4,39 & 392,13 & 78,58 & 30,74 & 2,55 & 51,46 & 1,61 & 1,25 & 2,55 \\
\hline Norme & $\begin{array}{c}+, 0,- \\
\text { Yn }\end{array}$ & 3,75 & 0,75 & 10 & 100 & 100 & 30 & 1 & 50 & 0,75 & 0,5 & 1 \\
\hline
\end{tabular}

\begin{tabular}{|c|c|c|c|r|r|r|r|}
\hline Y30 & Y39 & Y40 & Y41 & Y42 & Y43 & \multicolumn{1}{|c|}{ Y44 } & \multicolumn{1}{c|}{ Y45 } \\
\hline 15372,38 & 7,71 & 23,13 & 3,55 & 7,85 & 6,70 & 14,41 & 49,55 \\
\hline 5000 & 1 & 22,75 & 3,75 & 2 & 1,5 & 15 & 50 \\
\hline
\end{tabular}

10. Service civique d'aide à l'emploi et au développement (SCAED)

\begin{tabular}{|c|c|c|c|c|c|r|r|r|r|r|r|r|}
\hline ICH & Y13 & Y14 & Y15 & Y16 & Y6 & Y19 & Y20 & Y22 & Y24 & Y25 & Y26 & Y28 \\
\hline $\begin{array}{c}\text { SCAE } \\
\text { DY17 }\end{array}$ & $+75,00$ & 3,85 & 0,91 & 6,46 & 360,68 & 98,25 & 30,81 & 1,85 & 91,15 & 1,79 & 2,55 & 3,26 \\
\hline Norme & $\begin{array}{c}+, 0,- \\
\text { Yn }\end{array}$ & 3,75 & 0,75 & 10 & 100 & 100 & 30 & 1 & 50 & 0,75 & 0,5 & 1 \\
\hline
\end{tabular}

\begin{tabular}{|c|c|c|r|r|r|r|r|}
\hline Y30 & Y39 & Y40 & Y41 & \multicolumn{1}{c|}{ Y42 } & \multicolumn{1}{c|}{ Y43 } & \multicolumn{1}{c|}{ Y44 } & \multicolumn{1}{c|}{ Y45 } \\
\hline 11614,46 & 3,43 & 23,58 & 3,45 & 5,80 & 5,07 & 14,44 & 48,55 \\
\hline 5000 & 1 & 22,75 & 3,75 & 2 & 1,5 & 15 & 50 \\
\hline
\end{tabular}

\section{Programme d'Appui à l'Amélioration de l'Employabilité et à l'Insertion des Jeunes (PAAEIJ) volet THIMO}

\begin{tabular}{|c|c|r|r|r|r|r|r|r|r|r|r|r|}
\hline ICH & Y13 & Y14 & Y15 & Y16 & Y6 & Y19 & Y20 & Y22 & Y24 & Y25 & Y26 & Y28 \\
\hline $\begin{array}{c}\text { P2AEIJ } \\
\text { Y19 }\end{array}$ & $+56,25$ & 3,82 & 0,95 & 3,56 & 417,39 & 75,89 & 30,58 & 1,25 & 61,64 & 1,34 & 1,58 & 3,45 \\
\hline Norme & $\begin{array}{c}+, 0,- \\
\text { Yn }\end{array}$ & 3,75 & 0,75 & 10 & 100 & 100 & 30 & 1 & 50 & 0,75 & 0,5 & 1 \\
\hline
\end{tabular}

\begin{tabular}{|c|c|c|c|r|r|r|r|}
\hline Y30 & Y39 & Y40 & Y41 & \multicolumn{1}{c|}{ Y42 } & \multicolumn{1}{c|}{ Y43 } & \multicolumn{1}{c|}{ Y44 } & \multicolumn{1}{c|}{ Y45 } \\
\hline 15944,99 & 8,14 & 23,01 & 3,58 & 7,90 & 6,91 & 14,34 & 48,95 \\
\hline 5000 & 1 & 22,75 & 3,75 & 2 & 1,5 & 15 & 50 \\
\hline
\end{tabular}




\section{Contrat Désendettement Développement volet Emploi (C2D Emploi)}

\begin{tabular}{|c|c|c|c|c|c|c|c|c|c|c|c|c|}
\hline $\mathrm{ICH}$ & Y13 & Y14 & Y15 & Y16 & Y6 & Y19 & Y20 & Y22 & Y24 & Y25 & Y26 & Y28 \\
\hline DDAO & 3,82 & 0,93 & 4,45 & 355,45 & 100,27 & 30,56 & 2,69 & 156,13 & 1,43 & 0,86 & 1,95 & 3,82 \\
\hline Norme & $\begin{array}{c}+, 0,- \\
\text { Yn }\end{array}$ & 3,75 & 0,75 & 10 & 100 & 100 & 30 & 1 & 50 & 0,75 & 0,5 & 1 \\
\hline
\end{tabular}

\begin{tabular}{|r|r|r|r|r|r|r|r|}
\hline \multicolumn{1}{|c|}{ Y30 } & \multicolumn{1}{c|}{ Y39 } & \multicolumn{1}{c|}{ Y40 } & \multicolumn{1}{c|}{ Y41 } & \multicolumn{1}{c|}{ Y42 } & \multicolumn{1}{c|}{ Y43 } & \multicolumn{1}{c|}{ Y44 } & \multicolumn{1}{c|}{ Y45 } \\
\hline 13483,65 & 6,86 & 22,99 & 2,88 & 6,67 & 5,84 & 14,32 & 37,93 \\
\hline 5000 & 1 & 22,75 & 3,75 & 2 & 1,5 & 15 & 50 \\
\hline
\end{tabular}

\section{Agir pour les jeunes}

\begin{tabular}{|c|c|r|r|r|r|r|r|r|r|r|r|r|}
\hline ICH & Y13 & Y14 & Y15 & Y16 & Y6 & Y19 & Y20 & Y22 & Y24 & Y25 & Y26 & Y28 \\
\hline APJY21 & $+60,00$ & 3,01 & 0,81 & 9,63 & 74,51 & 95,55 & 24,06 & 1,58 & 44,74 & 1,25 & 1,55 & 2,68 \\
\hline Norme & $\begin{array}{c}+, 0,- \\
\text { Yn }\end{array}$ & 3,75 & 0,75 & 10 & 100 & 100 & 30 & 1 & 50 & 0,75 & 0,5 & 1 \\
\hline
\end{tabular}

\begin{tabular}{|c|c|c|c|r|r|r|r|}
\hline Y30 & Y39 & Y40 & Y41 & Y42 & Y43 & \multicolumn{1}{c|}{ Y44 } & \multicolumn{1}{c|}{ Y45 } \\
\hline 6230,98 & 1,71 & 18,19 & 3,58 & 2,43 & 2,13 & 11,28 & 48,55 \\
\hline 5000 & 1 & 22,75 & 3,75 & 2 & 1,5 & 15 & 50 \\
\hline
\end{tabular}

